\section{Loop diuretic use increases bone loss in older men}

Loop diuretics are commonly prescribed to older adults to induce diuresis in fluid-overload states, such as congestive heart failure, and to treat hypertension; however, these agents increase urinary calcium excretion and have been associated with an increased risk of osteoporotic fracture in observational studies and with bone loss in postmenopausal women. Lim et al. have now found that long-term use of loop diuretics is associated with increased rates of hip bone loss in older men.

The authors examined data from 3,269 US men aged $\geq 65$ years prospectively enrolled in the Osteoporotic Fractures in Men study. At baseline and at follow-up (average 4.6 years), bone mineral density at the hip was assessed by dual energy X-ray absorptiometry, and use of loop diuretics-including furosemide, bumetanide and torsemide-was ascertained.

After multivariate adjustment for confounding factors such as age, BMI and cardiovascular health, the average annual rate of total bone loss at the hip among men who were using loop diuretics both at baseline and at followup $(n=84)$ was approximately 2.5 -fold greater than in nonusers $(n=3,004)$ and approximately 1.5 -fold greater than in those who were using loop diuretics either at baseline or at followup $(n=181)$. Similar differences between the groups were seen for rates of hip bone loss at the femoral neck and trochanter.

The authors recommend that clinicians consider the possibility of future bone loss when prescribing loop diuretics to older men.

Original article Lim LS et al. (2008) Loop diuretic use and increased rates of hip bone loss in older men: the Osteoporotic Fractures in Men Study. Arch Intern Med 168: 735-740

\section{Point-of-care measurement device can accurately identify patients with microalbuminuria}

Although a number of desktop systems allow clinicians to measure urinary albumin excretion directly at the point of care, it is not clear whether these devices are as precise and accurate as central laboratory measurements. To evaluate the performance of one such system - the HemoCue Albumin 201 Analyzer, HemoCue, Ängelholm, Sweden-in a cohort of patients, Lambers Heerspink et al. compared the results with those obtained by nephelometry at a central laboratory.

Among the 259 first morning void samples obtained, nephelometry indicated that 151 had albumin concentrations within the measuring range of the HemoCue system; these samples were used for analysis. The coefficient of variation of 10 consecutive HemoCue measurements was in the range 4.9-8.0\%, meeting the FDA precision criterion for introduction of a new laboratory method. In total, $94 \%$ of the HemoCue measurements differed from the nephelometer measurements by $\leq 10 \mathrm{mg} / \mathrm{l}$ or $\leq 30 \%$, just under the FDA accuracy cut-off of $95 \%$. Analysis of receiver operating characteristic curves revealed that the sensitivity of the two types of test for predicting albuminuria of $\geq 30 \mathrm{mg}$ in a subsequent $24 \mathrm{~h}$ urine collection on the basis of the albumin concentration of a first morning void were identical at the clinically used cut-off value of $20 \mathrm{mg} / \mathrm{l}$. Among individuals with albuminuria ( $24 \mathrm{~h}$ urinary albumin excretion $\geq 30 \mathrm{mg} / 24 \mathrm{~h}$ ), the median intraindividual coefficients of variation of urinary albumin concentration measured by the two tests were similar.

The authors conclude that the HemoCue system is a valid alternative to central laboratory analysis for the identification and monitoring of patients with microalbuminuria.

Original article Lambers Heerspink HJ et al. (2008)

Screening and monitoring for albuminuria: the performance of the HemoCue point-of-care system. Kidney Int [doi:10.1038/ki.2008.186]

\section{Serum cystatin $C$ versus serum creatinine for GFR estimation in patients with HIV}

In patients with HIV, many of whom have liver disease or decreased lean body mass, creatininebased estimated glomerular filtration rates (eGFRs) might overestimate kidney function. Jones et al. have compared creatinine-based and cystatin-C-based eGFRs in patients with HIV and have determined that cystatin-C-based eGFR could be a more reliable marker of chronic kidney disease in this population.

Serum creatinine and cystatin $C$ levels were measured in 250 US patients with HIV who were on highly active antiretroviral therapy. The prevalence of abnormal kidney function, 